\title{
Mechanical Differences between Barbell and Body Optimum Power Loads in the Jump Squat Exercise
}

\author{
by \\ Irineu Loturco ${ }^{1,2}$, Lucas A. Pereira ${ }^{1}$, Vinicius Zanetti ${ }^{3}$, Katia Kitamura ${ }^{1}$ \\ César C. Cal Abad1, Ronaldo Kobal' ${ }^{1}$, Fabio Y. Nakamura ${ }^{1,4}$
}

This study compared the values of bar-peak force $\left(P F_{B a r}\right)$ and power $\left(P P_{B a r}\right)$, body-peak force $\left(P F_{B o d y}\right)$ and power $\left(P P_{B o d y}\right)$ and bar-mean propulsive power (MPPBar) in different jump-squat (JS) conditions: unloaded condition (UC); bar-loaded condition (BLC) and optimum bar-MPP condition (OBC). Twenty-five soccer players performed the JS using a bar with negligible mass (UC), using the Smith-machine bar (BLC) and using the load capable of maximizing the bar-MPP $(\mathrm{OBC})$. The PFBody was significantly higher in the UC $(2847.9 \pm 489.1 \mathrm{~N})$ than in the OBC $(2655.4 \pm$ $444.3 \mathrm{~N})$. The UC presented greater PPBody $(3775.9 \pm 631.5 \mathrm{~W})$ than the BLC $(3359.7 \pm 664.3 \mathrm{~W})$ and $O B C(3357.8 \pm$ $625.3 \mathrm{~W})$. The OBC presented higher values of $P F_{B a r}, P P_{B a r}$ and $M P P_{B a r}(676.2 \pm 109.4 \mathrm{~W})$ than the BLC $\left(M P P_{B a r}=\right.$ $425.8 \pm 53.7 \mathrm{~W}$ ) (all $p<0.05)$. In the OBC (compared to the UC), the body peak-power presented a reduction of $\approx 11 \%$, while generating bar-power output from $\approx 59$ to $73 \%$ higher than the BLC. While the fact that the body-peak power is maximized in the UC denotes a mechanical phenomenon, the bar-optimum load represents an intensity at which both components of the power equation (force and velocity) are optimized. This has important implications for sports training.

Key words: optimal loads, muscle power, ballistic, plyometrics, neuromuscular.

\section{Introduction}

In the literature, the load capable of maximizing the muscle power production in a specific strength-power exercise is usually defined as the optimum power load (OPL) (Cormie et al., 2011; Loturco et al., 2013). For some authors, due to its optimized mechanical characteristics, this specific range of loads may provide effective neuromuscular training stimuli to improve strength-power performance, both in recreational and elite athletes (Cormie et al., 2011). Nevertheless, in spite of its suggested effectiveness (Cormie et al., 2011), the determination of the OPL is somewhat complicated, making the selection of appropriate training strategies difficult for coaches. Indeed, the use of different methods and measures to define the optimum range of loads has precluded drawing definitive conclusions on this issue (Cormie et al., 2011; Mcmaster et al., 2014).

It has been common to compare the bodypower output collected in unloaded jump squats (JS) (i.e., JS at $0 \%$ of the squat one repetition maximum [1-RM]) with body-power assessed in loaded jump squats (Cormie et al., 2011; JimenezReyes et al., 2015). For instance, Jimenez-Reyes et al. (2015) found that the load that maximized JS

\footnotetext{
1 - NAR - Nucleus of High Performance in Sport, São Paulo, SP, Brazil.

2 - Department of Mechatronics Engineering, University of São Paulo, SP, Brazil.

3 - Red Bull Brazil Football, SP, Brazil.

4 - State University of Londrina, Londrina, PR, Brazil.
} 
power output was $0 \%$ of $1-\mathrm{RM}$, when comparing unloaded countermovement jumps with JSs executed under different loading intensities. Similarly, Cormie et al. (2007b) reported maximum values of body muscle power in unloaded JSs, after examining the values of peak power produced within a range of loads from zero to $90 \%$ of maximal dynamic strength, calculated by including or not including the contribution of body mass in force output. Thus, it is clear from the literature that body-power in the JS is optimized in the complete absence of external loads (i.e., $0 \%$ of 1-RM) (Cormie et al., 2007c; Nuzzo et al., 2010).

Another common way of testing the "optimum power zone" is to assess the power production directly from the barbell, using a linear position transducer (LPT) (Loturco et al., 2015d; Talpey et al., 2014). Different from the body-power measurements (traditionally performed using force plates) (Soriano et al., 2015), the bar-power production is always optimized at a moderate range of loads (Loturco et al., 2015d; Loturco et al., 2013). For instance, Baker et al. (2001) revealed that loads in the range of circa $48-63 \%$ of $1-\mathrm{RM}$ seemed to be effective at maximizing bar-power during the JS in powertrained subjects. Importantly, maximum power tends to be lower in non-specialized athletes, as a consequence, the range of loads used to optimize the bar-power production in this case may need to be lower. Indeed, Cormie et al. (2007a) reported that values of peak force and peak power were overestimated in recreationally trained subjects when executing the JS at 30\% of 1-RM.

Recently, some authors have suggested the use of mean propulsive power (MPP) to determine the optimum power zone in strengthpower exercises (Loturco et al., 2015d; SanchezMedina et al., 2010). Accordingly, SanchezMedina et al. (2010) demonstrated that the mechanical values collected during the propulsive phase of a lift better reflected the differences in neuromuscular performance between two given individuals. In fact, JS MPP has been shown to be highly correlated with specific performance in athletes from distinct sports (Loturco et al., 2014, 2015a, 2016a). Additionally, it has been shown that training at the optimum load produces greater performance improvements than traditional strength training and reduces the speed-power decrements that usually occur during soccer preseasons (Loturco et al., 2015d, 2016b).

Given the effectiveness of MPP, it is important to determine the mechanical behaviour of body- and bar-peak power in the MPP optimum power zone. Based on it, strength coaches could select better and more appropriate training strategies to increase strength-power abilities in athletes. Furthermore, this knowledge may clarify the conceptual confusion that exists in the literature concerning the estimation of optimum power loads. Therefore, this study aimed at examining the mechanical behaviour of body- and bar-peak power in the JS MPP optimum power zone. For this purpose, we investigated the values of bar-peak force and power, body-peak force and power and bar-mean propulsive power in three different conditions: (1) unloaded condition (UC); (2) bar-loaded condition (BLC); and (3) optimum bar-MPP condition (OBC).

\section{Material and Methods}

Study Design

This cross-sectional study compared the mechanical output (peak force, peak power and mean propulsive power) obtained using a linear position encoder and a force plate in the unloaded jump squat, the bar-loaded jump squat and the jump squat at the optimum power load. This conceptual investigation was originally designed to differentiate the mechanical behaviour of exercising using or not using external loading during the jump squat, and explore the possibilities of contrasting the body- and bardynamic and kinematic output. Prior to the testing session, a general and specific warm-up was performed, involving light running ( $5 \mathrm{~min}$ at a self-selected pace followed by $3 \mathrm{~min}$ lower limb active stretching) and submaximal attempts of each testing exercise (e.g., submaximal vertical or horizontal jumps).

\section{Participants}

Twenty-five well trained youth soccer players (18.5 \pm 0.4 years; $71.3 \pm 8.5 \mathrm{~kg} ; 176.2 \pm 7.1$ $\mathrm{cm}$ ) volunteered to participate in the study. Due to their professional training and assessment routine, all players were highly familiarized with the testing procedures. The athletes were members of the same soccer club and were in the 
competitive period, having recently participated in the most important regional youth soccer competition in Brazil (i.e., Under-20 São Paulo State Championship). The soccer players and their legal guardians (for the athletes $<18$ years) were informed of the study procedures and signed an informed consent form prior to the investigation. The study was approved by the Bandeirante Anhanguera University Ethics Committee.

\section{Jump Squat Tests}

The JS tests comprised sets of 4 repetitions at maximal velocity for each load, with $15 \mathrm{~s}$ rest intervals between repetitions. The first set was performed in the "unloaded condition" (UC) $(0 \%$ of 1-RM), set up with participants in a standing position while holding a plastic bar with negligible mass across their shoulders (Figure 1) in order to mimic the movement pattern performed in the actual loaded conditions. The athletes executed a knee flexion until the thigh was parallel to the ground $\left(\approx 100^{\circ}\right.$ knee angle) and, after a command, jumped as fast as possible, without their shoulder losing contact with the bar. Considering that JSs are always executed with (at least) the barbell (Soriano et al., 2015), the following set was performed solely using the Smith-machine bar, which weighs $20 \mathrm{~kg}$ (fixed bar-loaded condition - BLC). The BLC was intentionally created to establish the mechanical behaviour of body- and bar-peak power in this minimum loading condition (Figure 2). Afterwards, the soccer players performed the JS with a load corresponding to $40 \%$ of their body mass (BM). A load of $10 \%$ of BM was gradually added in each set, until a decrease in MPP was observed. The individual maximum MPP value obtained (optimum bar-MPP condition - OBC) was considered for further analysis. A 5 min rest interval was allowed between sets. In all sets and repetitions, the JS was performed with the subjects positioned on the force plate (AccuPower, AMTI, USA). Similarly, except for the UC, all the repetitions corresponding to the BLC and OBC were performed with the bar attached to a linear position transducer (T-Force, Dynamic Measurement System; Ergotech Consulting S.L., Murcia, Spain).

\section{Bar Mechanical Measures}

To determine peak power ( $\mathrm{PP}_{\mathrm{Bar}}$ ), peak force $(\mathrm{PF} B a r)$ and mean propulsive power (MPP), a linear position transducer (T-Force, Dynamic
Measurement System; Ergotech Consulting S.L., Murcia, Spain) was used, attached to the Smithmachine bar. The finite differentiation technique was applied to calculate bar velocity and acceleration. The bar position data were sampled at $1,000 \mathrm{~Hz}$ using a PC (Toshiba Satellite; Toshiba Computers, Tokyo, Japan). Mean rather than peak power was used as Sanchez-Medina et al. (2010) had observed that the mean mechanical values during the propulsive phase better reflected the differences in neuromuscular potential between individuals. Such an approach permits to avoid underestimation of the true strength potential, as the higher the mean velocity (and the lower the relative load), the greater the relative contribution of the braking phase to the entire concentric time.

\section{Force Plate Measures}

Peak power (PPBody) and peak force $\left(\mathrm{PF}_{\text {Body }}\right)$ were assessed during the JS tests on a force plate (AccuPower, AMTI, USA) with custom-designed software (AccuPower; AMTI, Watertown, MA, USA), which sampled at a rate of $400 \mathrm{~Hz}$ (Walsh et al., 2006).

\section{Statistical Analysis}

Means and standard deviations (SD) were used to represent centrality and spread of the data. A repeated measures analysis of variance was used to compare force and power variables between the three conditions (UC, BLC and OBC), followed by the Bonferroni post hoc test in order to identify where the differences occurred. The effect size (ES) was also calculated by the mean difference between a pair of condition values divided by their mean standard deviation (Cohen, 1988). The magnitude of the ES was interpreted using the thresholds proposed by Rhea (2004) as follows: $<0.25,0.25-0.50,0.50-1$, and $>1$ for trivial, small, moderate and large, respectively. The level of significance was set at $p<0.05$.

\section{Results}

The maximum MPP values in the $\mathrm{OBC}$ were observed in a load corresponding to $\approx 68 \%$ of BM (values ranging from 60 to $70 \%$ ). Table 1 shows the comparisons between body and bar mechanical output (peak force, peak power and mean propulsive power) in the three different JS conditions (unloaded, bar-loaded and optimum bar-MPP condition). The PFBody was significantly higher in the UC than in the OBC (difference: $6.76 \%$; ES $=0.41$, small; $p<0.05$ ) and was not 
significantly different from the BLC (difference: $5.16 \%$; ES $=0.31$, small; $p>0.05$ ). The UC presented greater PPBody than the BLC and OBC (difference: $11.02 \%$; $\mathrm{ES}=0.64$, moderate $; p<0.05$; difference: $11.07 \%$; $\mathrm{ES}=0.67$, moderate; $p<0.05$, respectively). In addition, $\mathrm{PF}_{\text {Body }}$ and PPBody were not significantly different between BLC and OBC (difference: $1.68 \%$; $\mathrm{ES}=0.10$, trivial; $p>0.05$; difference: $0.05 \%$; $\mathrm{ES}=0.01$, trivial; $p>0.05$, respectively). In relation to the bar measures, when compared to the BLC, the OBC presented higher values in the three assessed variables (PFBar, $\mathrm{PP}_{\text {Bar }}$ and MPPBar) (difference: 92.56\%; ES = 4.92, large; $p<0.05$; difference: $72.60 \%$; $\mathrm{ES}=2.79$, large; $p<0.05$, difference: $58.82 \%$; ES $=3.07$, large; $p$ $<0.05$, respectively).

Figure 1

Athlete executing a jump squat in the unloaded condition. The exercise was performed using a plastic bar with negligible mass.

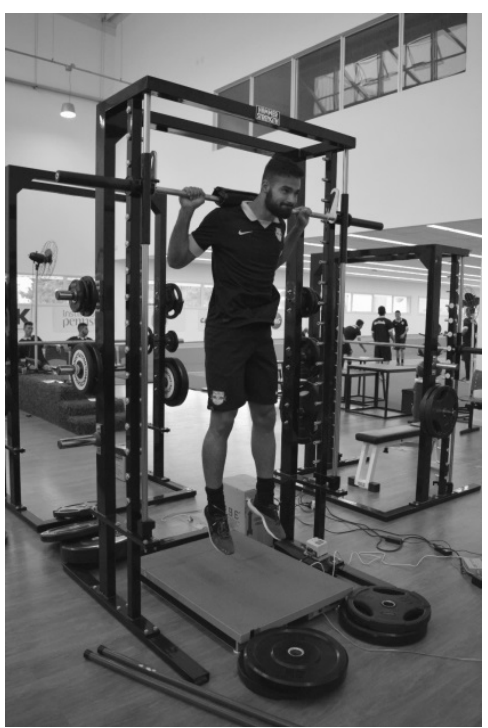

\section{Figure 2}

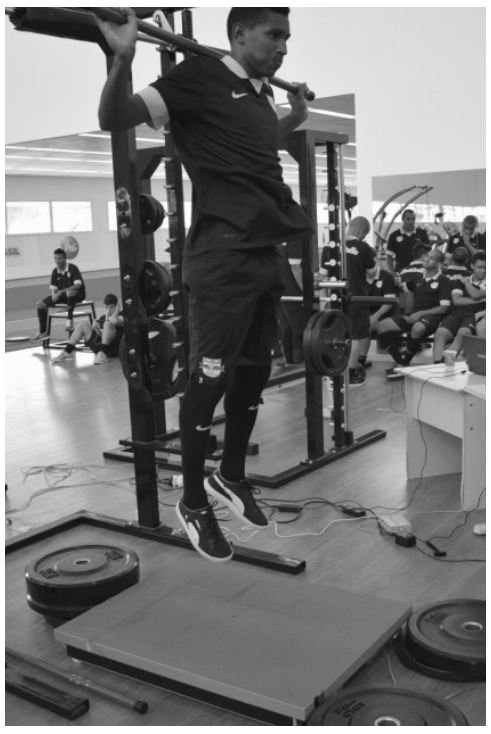

Athlete executing a jump squat in the bar-loaded condition, using only the Smithmachine barbell as the external load. In the optimum bar-mean propulsive power condition, a load capable of maximizing the individual's value of mean propulsive power was added to the Smith-machine barbell. 
Table 1

Comparisons of the body and bar mechanical measures between the three loading conditions.

\begin{tabular}{lccc} 
& UC & BLC & OBC \\
\hline PFBody $_{\text {(N) }}$ & $2847.9 \pm 489.1^{*}$ & $2700.8 \pm 448.2$ & $2655.4 \pm 444.3$ \\
PPBody $_{\text {(W) }}$ & $3775.9 \pm 631.5^{* *}$ & $3359.7 \pm 664.3$ & $3357.8 \pm 625.3$ \\
PFBar $_{\text {(N) }}$ & - & $416.4 \pm 35.1^{*}$ & $801.9 \pm 121.6$ \\
PPBar $_{\text {(W) }}$ & - & $938.1 \pm 160.1^{*}$ & $1619.2 \pm 328.1$ \\
MPPBar (W) & - & $425.8 \pm 53.7^{*}$ & $676.2 \pm 109.4$ \\
\hline
\end{tabular}

$U C=$ unloaded condition; $B L C=$ bar loaded condition; $O B C=$ optimum bar-MPP condition; $P F_{B o d y}=$ peak force measured on the force plate; $P P_{B o d y}=$ peak power measured on the force plate; $P F_{B a r}=$ peak force measured in the bar; $P P_{B a r}=$ peak power measured in the bar; $M P P_{B a r}=$ mean propulsive power measured in the bar; ${ }^{*} p<0.05$ in comparison to the $O B C ; " * p<0.05$ in comparison to the BLC.
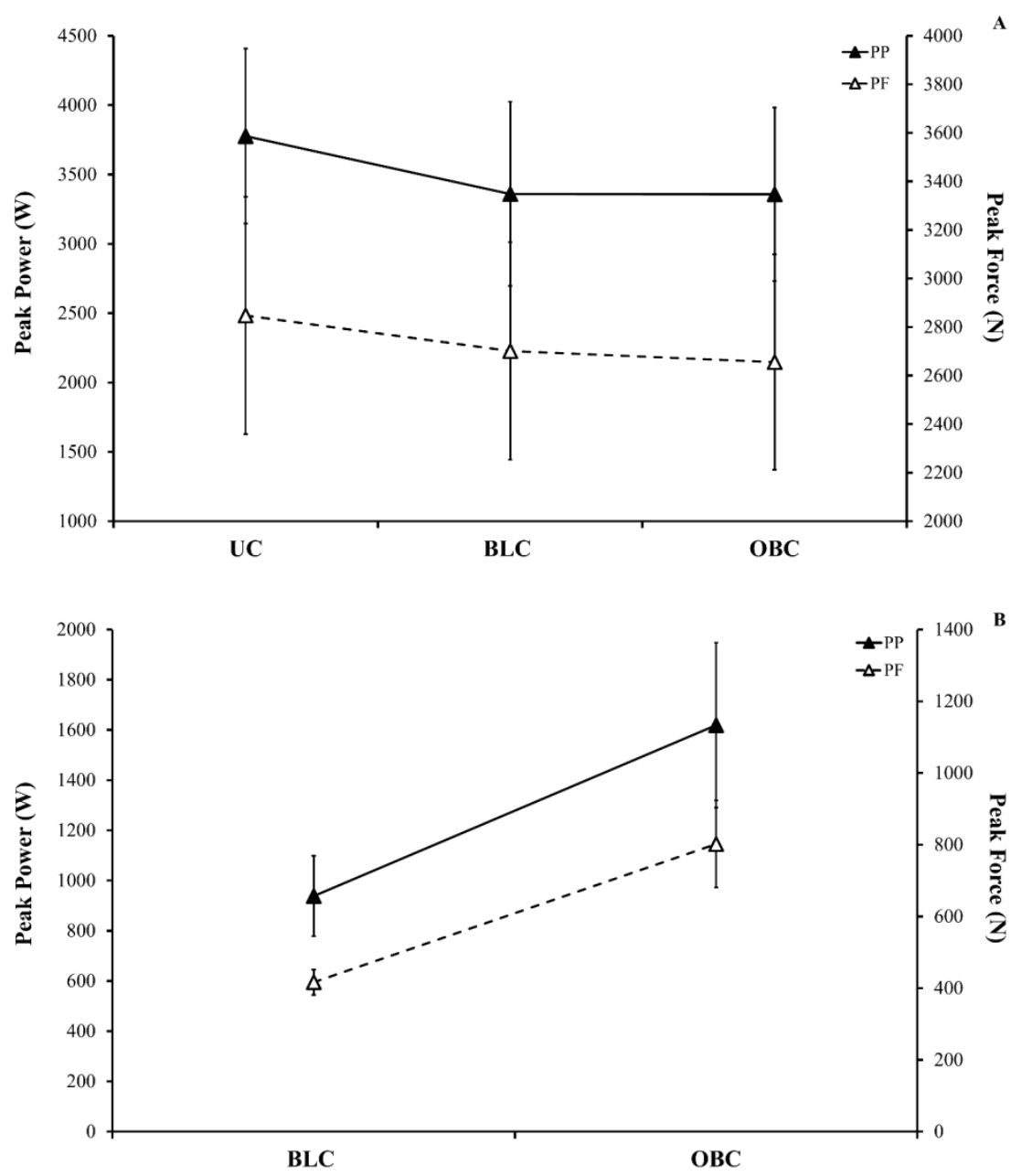

Figure 3

Body (Panel $A$ ) and bar (Panel B) mechanical measures in different loading conditions. 
Figure 3 depicts the PF and PP variations in the three different loading conditions in order to present the mechanical behaviour of body- and bar-mechanical output among the three different conditions of JS execution.

\section{Discussion}

This conceptual research is the first study to test and compare the mechanical behaviour of three distinct values of muscle power (i.e., barand body-peak power and bar-mean propulsive power) in three different conditions: UC, BLC and OBC. As already reported in other studies (Cormie et al., 2007c; Nuzzo et al., 2010), we observed that the maximum values of body-peak power occurred in the complete absence of external loads (i.e., UC). Importantly, when compared with both JS loaded settings (i.e., BLC and OBS); this theoretically superior condition of generating muscle power was only able to increase body-peak power by $11 \%$ (ES $\approx 0.65$ ). Furthermore, using a zero-loaded barbell, the UC was not capable of providing any external resistance to the subjects while jump squatting. Finally, in line with our hypothesis, independently of the power measure considered (i.e., PPBar or MPPBar), the OBC was able to generate bar-power output from $\approx 59$ to $73 \%$ higher than the BLC.

Our results are in agreement with other investigations reporting that JS body-peak power is maximized in the UC (Cormie et al., 2011). Remarkably, to draw this conclusion, the authors had to create an artificial training condition, by instructing their subjects to hold a PVC pipe or a $0.4 \mathrm{~kg}$ carbon fiber pole (in place of a barbell) (Mcbride et al., 2011), to enable them to perform JSs at $0 \%$ 1-RM. More recently, another study revealed that body-peak power in the JS was reached with a load corresponding to $0 \% 1-\mathrm{RM}$, after comparing the power output obtained in a loaded JS with the measures collected in unloaded countermovement jumps (Jimenez-Reyes et al., 2015), which is actually a different exercise. Essentially, as defined by Soriano et al. (2015), during the JSs, the subjects have to set up in a standing position while holding a barbell across their shoulders. Considering that the Olympic barbells (used in JSs) weigh on average $20 \mathrm{~kg}$ (Cormie et al., 2010a), it seems worthless to create simulated conditions for training purposes (i.e., using barbells of negligible weight during testing sessions) only to optimize the body-power production (Dayne et al., 2011). In addition, elite athletes always execute JSs with appropriate weight plates, since it has been established that subjects who train with the load that maximizes mechanical power (i.e., loaded JSs at $30 \%$ of maximum isometric force) achieve best overall results in enhancing dynamic athletic performance (rather than performing traditional strength training or plyometric training) (Mcbride et al., 2002; Wilson et al., 1993).

In fact, the simulated unloaded JS only mimics the widely used squat jump - a type of a vertical jump generally used to evaluate jumping performance (Loturco et al., 2015c, 2016a). Similarly, unloaded countermovement jumps are normally employed to assess vertical jump height and in training sessions aimed at developing plyometric ability in trained subjects (Loturco et al., 2015c). Thus, although body-peak power tends to be maximized in the UC, under this mechanical condition the training stimulus is comparable to that found in regular plyometric training, which potentially reduces the importance of this conceptual determination. Even recognizing that plyometrics can improve neuromuscular function in elite athletes (Holcomb et al., 1996; Saez-Saez De Villarreal et al., 2010), it is essential to underline that loaded jumps have already shown their greater effectiveness in enhancing the overall athletic performance (Cormie et al., 2011; Wilson et al., 1993).

In this study, the BLC setting was deliberately designed to establish (and mediate) the mechanical behaviour of body- and bar-peak power while executing the JS at the minimum possible load (i.e., using exclusively the $20 \mathrm{~kg}$ barbell). Taking into account that JSs are always performed with the barbell (Soriano et al., 2015), this realistic approach enables coaches and sport scientists to better understand the importance of measuring muscle power output directly from the barbell. In spite of the slight decrease in bodypeak power in the BLC $(\approx-11 \%$, in comparison to the UC), in this condition the subjects can jump with a load of $20 \mathrm{~kg}$, thus producing significant bar-peak power of $\approx 940 \mathrm{~W}$. Furthermore, when jumping with this minimum external load, the subjects can generate important peak force of $\approx$ $416 \mathrm{~N}$ on the barbell. In this sense, it is clear from 
the literature that weight-resistance exercises (i.e., exercises with external loads) are essential to properly develop strength-power qualities and elicit neuromuscular adaptations in recreational and elite athletes (Hansen and Cronin, 2009). Moreover, rather than comparing maximum mechanical outcome which can be produced under different loaded-conditions, defining baroptimum power zones implies searching for the best combination of both components of the power equation. Based on this mechanical relation, it is possible to determine a specific range of loads capable of maximizing the bar-force production without significant impairment of movement velocity, and thus enhancing barpower output.

Concisely, this optimum range of loads solely represents balanced and optimized integration between force and velocity in a determined mechanical system. However, when selecting the optimum bar-loads to perform JSs, sport practitioners consider not only the resistance placed on the barbell, but also the relationship between this referred mass and bar velocity. As the athletes regularly have to apply great amounts of force at high velocities, it is plausible that this individually-based loading selection pattern may be more interrelated to sports performance than patterns based solely on the mass calculation (i.e., fixed $\%$ of 1-RM) (Loturco et al., 2015b). Importantly, as already mentioned, the possibility of selecting mean mechanical values related to the propulsive phase of a lift (i.e., MPP) may increase the importance of MPP in sports performance settings.

It is critical to observe that, in many motor tasks, the athletes have to apply force/develop power while working (only) against their own body mass (Loturco et al., 2014, 2015c). Nevertheless, several studies have already reported that bar MPP in the OBC is highly related to actual performance in different sport disciplines (Loturco et al., 2014, 2015a, 2016a). In addition, it has been demonstrated that training at this optimum intensity may lead to superior performance improvements when compared to other loading/unloading conditions (Loturco et al., 2016b; Moss et al., 1997; Wilson et al., 1993). Although the reasons for these significant findings are not entirely clear, the current study shows that this range of loads (i.e., $\mathrm{OBC}$ ) presents a singular mechanical condition. Firstly, during the OBC (in relation to the BLC) bar-peak power increases by $\approx 73 \%$ and bar-MPP increases by $\approx$ $59 \%$. Secondly, in spite of the reduction in movement velocity/acceleration (due to the loading effect), in the $\mathrm{OBC}$, body-peak power and body-peak force presented only slight reductions of $\approx 11 \%$ and $\approx 7 \%$, respectively (in relation to the UC). Indeed, the parametric relationship between force and velocity (i.e., the higher the force, the lower the velocity) determines this enhanced mechanical condition in the OBC (Loturco et al., $2015 b)$. It appears that at this optimum range of loads, force is optimized, although velocity is not heavily impaired.

In fact, this force optimization is, unquestionably, the key difference between the UC and OBC. Although body acceleration necessarily diminishes under loaded conditions, in the $\mathrm{OBC}$, in comparison to the $\mathrm{UC}$, body peakforce presented only a small reduction of $\approx 11 \%$. However, due to the complete absence of external loads, in the UC, the athletes cannot apply any quantity of force against any type of implement. Assuming that overloading is an important premise of strength-power development (Cormie et al., 2010b; Hansen and Cronin, 2009), the UC is inherently limited to improving specific neuromuscular performance, mainly in highly trained subjects (Baker et al., 2001). Conversely, when jumping in the $\mathrm{OBC}$, the athletes can apply significant amounts of bar-peak force. Of note, in this study, the subjects were able to generate peak force of $\approx 800 \mathrm{~N}$ on the barbell in the OBC. Certainly, training under this dynamic condition produces greater adaptations (than the UC) in power-related performance (Cormie et al., 2010b).

We acknowledge the inherent limitations of a cross-sectional study design, which reduces our ability to confidently infer causality. However, it is important to emphasize that the effects of training under loaded or unloaded conditions are well-established in the scientific literature, and this study was exclusively conceived to better elucidate the conceptual basis of these constructs. Hence, from a biomechanical perspective, there is no point in comparing bodyand bar-peak power. The scientific concept behind this theme involves much more than a single mechanical comparison. When seeking for baroptimum power loads, coaches and sport 
scientists search for a more effective range of loads to develop strength-power qualities in athletes. In opposition to the conventional loading method (i.e., \% of 1-RM), based on the scalar variable (i.e., mass) of the force equation, the optimum range of loads reflects, at the same time, the force and velocity applied to the barbell. This mechanical behaviour definitely seems to be directly related to actual sports performance. On the other hand, the fact that body-peak power is maximized in the UC simply denotes a mechanical phenomenon, which does not necessarily imply a causative relationship (the amount of power generated vs. training results).

\section{Conclusions}

Since it has been widely established that maximum body-peak power always occurs in the
UC, less effort should be spent on replicating this information. Conversely, due to the strong relationship between bar-power loads and actual sports performance, more studies should be performed to establish (and define) optimum range of loads for different types of strengthpower exercises. Additionally, further studies are necessary to extend the findings related to adaptations provided by training in the baroptimum power zones and their effects on athletic performance. Based on the conceptual differences elucidated in this paper, sports practitioners may better understand the importance of performing loaded muscle power tests in recreational and elite athletes.

\section{References}

Baker D, Nance S, Moore M. The load that maximizes the average mechanical power output during jump squats in power-trained athletes. J Strength Cond Res, 2001; 15: 92-97

Cohen J. Statistical Power Analysis for the Behavioral Sciences. Hillsdale (NJ): Lawrence Erlbaum Associates; 1988

Cormie P, Deane R, Mcbride JM. Methodological concerns for determining power output in the jump squat. J Strength Cond Res, 2007a; 21: 424-430

Cormie P, Mcbride JM, Mccaulley GO. The influence of body mass on calculation of power during lowerbody resistance exercises. J Strength Cond Res, 2007b; 21: 1042-1049

Cormie P, Mccaulley GO, Triplett NT, Mcbride JM. Optimal loading for maximal power output during lower-body resistance exercises. Med Sci Sports Exerc, 2007c; 39: 340-349

Cormie P, Mcguigan MR, Newton RU. Influence of strength on magnitude and mechanisms of adaptation to power training. Med Sci Sports Exerc, 2010a; 42: 1566-1581

Cormie P, Mcguigan MR, Newton RU. Adaptations in athletic performance after ballistic power versus strength training. Med Sci Sports Exerc, 2010b; 42: 1582-1598

Cormie P, Mcguigan MR, Newton RU. Developing maximal neuromuscular power: part 2 - training considerations for improving maximal power production. Sports Med, 2011; 41: 125-146

Dayne AM, Mcbride JM, Nuzzo JL, Triplett NT, Skinner J, Burr A. Power output in the jump squat in adolescent male athletes. J Strength Cond Res, 2011; 25: 585-589

Hansen K, Cronin J. Training loads for the development of lower body muscular power during squatting movements. J Strength Cond Res, 2009; 31: 17-33

Holcomb WR, Lander JE, Rutland RM, Wilson GD. The effectiveness of a modified plyometric program on power and the vertical jump. J Strength Cond Res, 1996; 10: 89-92

Jimenez-Reyes P, Pareja-Blanco F, Balsalobre-Fernandez C, Cuadrado-Penafiel V, Ortega-Becerra MA, Gonzalez-Badillo JJ. Jump squat performance and its relationship with relative training intensity in 
high-level athletes. Int J Sports Physiol Perform, 2015; 10: 1036-1040

Loturco I, Artioli GG, Kobal R, Gil S, Franchini E. Predicting punching acceleration from selected strength and power variables in elite karate athletes: a multiple regression analysis. J Strength Cond Res, 2014; 28: $1826-1832$

Loturco I, Kobal R, Maldonado T, Piazzi AF, Bottino A, Kitamura K, Cal Abad CC, Pereira LA, Nakamura FY. Jump squat is more related to sprinting and jumping abilities than Olympic push press. Int J Sports Med, 2015a; In Press

Loturco I, Nakamura FY, Artioli GG, Kobal R, Kitamura K, Cal Abad CC, Cruz IF, Romano F, Pereira LA, Franchini E. Strength and power qualities are highly associated with punching impact in elite amateur boxers. J Strength Cond Res, 2016a; 30: 109-116

Loturco I, Nakamura FY, Kobal R, Gil S, Cal Abad CC, Cuniyochi R, Pereira LA, Roschel H. Training for Power and Speed: Effects of Increasing or Decreasing Jump-Squat Velocity in Elite Young Soccer Players. J Strength Cond Res, 2015b; 29: 2771-2779

Loturco I, Nakamura FY, Kobal R, Gil S, Pivetti B, Pereira LA, Roschel H. Traditional periodization versus optimum training load applied to soccer players: effects on neuromuscular abilities. Int J Sports Med, 2016b; In Press

Loturco I, Pereira LA, Cal Abad CC, D'angelo RA, Fernandes V, Kitamura K, Kobal R, Nakamura FY. Vertical and horizontal jump tests are strongly associated with competitive performance in 100-m dash events. J Strength Cond Res, 2015c; 29: 1966-1971

Loturco I, Pereira LA, Kobal R, Zanetti V, Gil S, Kitamura K, Abad CC, Nakamura FY. Half-squat or jump squat training under optimum power load conditions to counteract power and speed decrements in Brazilian elite soccer players during the preseason. J Sports Sci, 2015d; 33: 1283-1292

Loturco I, Ugrinowitsch C, Roschel H, Tricoli V, Gonzalez-Badillo JJ. Training at the optimum power zone produces similar performance improvements to traditional strength training. J Sports Sci Med, 2013; 12: 109-115

Mcbride JM, Haines TL, Kirby TJ. Effect of loading on peak power of the bar, body, and system during power cleans, squats, and jump squats. J Sports Sci, 2011; 29: 1215-1221

Mcbride JM, Triplett-Mcbride T, Davie A, Newton RU. The effect of heavy- vs. light-load jump squats on the development of strength, power, and speed. J Strength Cond Res, 2002; 16: 75-82

Mcmaster DT, Gill N, Cronin J, Mcguigan M. A brief review of strength and ballistic assessment methodologies in sport. Sports Med, 2014; 44: 603-623

Moss BM, Refsnes PE, Abildgaard A, Nicolaysen K, Jensen J. Effects of maximal effort strength training with different loads on dynamic strength, cross-sectional area, load-power and load-velocity relationships. Eur J Appl Physiol Occup Physiol, 1997; 75: 193-199

Nuzzo JL, Mcbride JM, Dayne AM, Israetel MA, Dumke CL, Triplett NT. Testing of the maximal dynamic output hypothesis in trained and untrained subjects. J Strength Cond Res, 2010; 24: 1269-1276

Rhea MR. Determining the magnitude of treatment effects in strength training research through the use of the effect size. J Strength Cond Res, 2004; 18: 918-920

Saez-Saez De Villarreal E, Requena B, Newton RU. Does plyometric training improve strength performance? A meta-analysis. J Sci Med Sport, 2010; 13: 513-522

Sanchez-Medina L, Perez CE, Gonzalez-Badillo JJ. Importance of the propulsive phase in strength assessment. Int J Sports Med, 2010; 31: 123-129

Soriano MA, Jimenez-Reyes P, Rhea MR, Marin PJ. The optimal load for maximal power production during lower-body resistance exercises: a meta-analysis. Sports Med, 2015; 45: 1191-1205 
Talpey SW, Young WB, Saunders N. The acute effects of conventional, complex, and contrast protocols on lower-body power. J Strength Cond Res, 2014; 28: 361-366

Walsh MS, Ford KR, Bangen KJ, Myer GD, Hewett TE. The validation of a portable force plate for measuring force-time data during jumping and landing tasks. J Strength Cond Res, 2006; 20: 730-734

Wilson GJ, Newton RU, Murphy AJ, Humphries BJ. The optimal training load for the development of dynamic athletic performance. Med Sci Sports Exerc, 1993; 25: 1279-1286

\section{Corresponding author:}

\section{Irineu Loturco}

NAR - Nucleus of High Performance in Sport.

Av. Padre José Maria, 555 - Santo Amaro, 04753-060 - São Paulo, SP, Brazil.

Phonel.: +55-11-3758-0918

E-mail: irineu.loturco@terra.com.br 